\title{
Iterative reconstruction using a pyramid-shaped basis function
}

\author{
K. Schmitt, Student Member, IEEE, F. Noo, Member, IEEE, J. Hornegger, K. Stierstorfer, and H. Schöndube
}

\begin{abstract}
In iterative reconstruction, it is common to represent the continuous image as a finite linear combination of basis functions. Popular basis functions include the B-splines and the blobs. It turns out that the $B$-spline of order 0 corresponds to nearest-neighbour interpolation, and its parallel-beam projections are piecewise linear functions. Also, the B-spline of order 1 corresponds to bilinear interpolation and its parallel-beam projections are piecewise cubic polynomials. This observation brings the question of whether there exists an intermediate basis function with parallel-beam projections taking the form of a piecewise quadractic polynomial. Here, we discuss image reconstruction using a pyramid-shaped basis function that satisfies this condition. It turns out that this function performs as well as the B-splines of order 1 in terms of bias and noise. Unfortunately, reconstruction with this basis function requires a correction method to remove an inconvenient grid pattern.
\end{abstract}

\section{INTRODUCTION}

In iterative reconstruction, it is common to represent the continuous image as a finite linear combination of basis functions. Popular basis functions include the B-splines [1] and the blobs [2]. Whereas the blobs are defined using three parameters together with the Kaiser-Bessel function, the Bsplines are simple piecewise polynomial functions defined by a single parameter: the degree, $n$, of the polynomial. It turns out that the B-spline of order $n=0$ corresponds to nearestneighbor interpolation, and its parallel-beam projections are piecewise linear functions. Also, the B-spline of order $n=1$ corresponds to bilinear interpolation and its parallel-beam projections are piecewise cubic polynomials. This observation brings the question of whether there exists an intermediate basis function with parallel-beam projections taking the form of a piecewise quadratic polynomial. We found that a pyramidshaped basis function satisfies this condition. Here, we give the definition of this function, we evaluate its Radon transform, and we investigate iterative reconstruction in X-ray CT using it.

Manuscript received May 5, 2012. This work was partially supported by a grant from Siemens AG, Healthcare Sector and by the U.S. National Institutes of Health (NIH) under Grant No. R01 EB007236. Its contents are solely the responsibility of the authors and do not necessarily represent the official views of the NIH.

Katharina Schmitt is a Ph.D candidate with the Chair of Pattern Recognition Lab, Friedrich-Alexander-University, Erlangen, Germany (e-mail: katharina.schmitt@studium.uni-erlangen.de). She is also with the Department of Radiology, the University of Utah, Salt Lake City, USA and with Siemens AG, Healthcare Sector, Forchheim, Germany.

Frédéric Noo is with the Department of Radiology, the University of Utah, Salt Lake City, USA (e-mail: noo@ucair.med.utah.edu).

Joachim Hornegger is with the Chair of Pattern Recognition, FriedrichAlexander-University of Erlangen-Nuremberg, Erlangen, Germany.

Karl Stierstorfer and Harald Schöndube are with Siemens AG, Healthcare Sector, Forchheim, Germany.
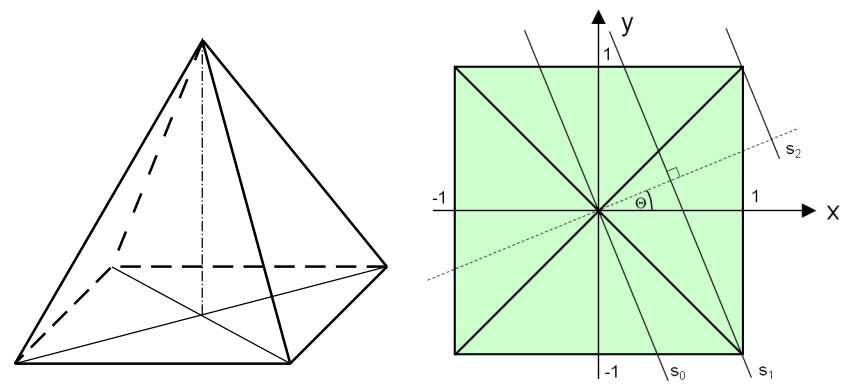

Fig. 1. (left) Pyramid-shaped basis function. (right) Top view of the pyramid together with the parallel-beam coordinates, $\theta$ and $s$.

\section{IMAGE REPRESENTATION}

Let $f(x, y)$ be the function describing the linear attenuation coefficient of X-rays as a function of the position within the field-of-view of the scanner. We represent $f$ by a linear combination of basis functions:

$$
f(x, y)=\sum_{k, l} c_{k l} \cdot b\left(\frac{x-x_{k}}{\Delta x}, \frac{y-y_{l}}{\Delta y}\right) .
$$

In this expression, the $c_{k l}$ are the basis function coefficients to be estimated, and the locations $x_{k}=k \Delta x$ and $y_{l}=l \Delta y$ are samples on a Cartesian grid with steps $\Delta x$ and $\Delta y$ in $x$ and $y$, respectively.

Naturally, the image quality of iterative reconstruction techniques depends on the choice of the basis functions. As discussed earlier, we consider here a pyramid-shaped basis function, defined as

$$
b(x, y)=\frac{3}{4} \cdot\left\{\begin{array}{lll}
1-|x| & \text { if } & |y| \leq|x| \\
1-|y| & \text { if } & |x| \leq|y| \\
0 & \text { if } & |x| \geq 1,|y| \geq 1
\end{array}\right.
$$

See Fig. 1 (left). This basis function is even in both $x$ and $y$ and invariant under rotations of 90 degrees. Therefore its Radon transform, $g(\theta, s)$, is fully specified by its values over the region $\{\theta \in[0, \pi / 4], \quad s \geq 0\}$. Using Fig. 1 (right), the expression for $g(\theta, s)$ over this region can be shown to be:

$$
g(\theta, s)=\frac{3}{4} \cdot \begin{cases}\frac{2 \cdot\left(s_{1} s_{2}-s^{2}\right)}{s_{1} s_{2}\left(s_{1}+s_{2}\right)} & \text { if } \quad 0 \leq s<s_{1} \\ \frac{2 \cdot\left(s_{2}-s\right)^{2}}{s_{2}\left(s_{2}^{2}-s_{1}^{2}\right)} & \text { if } \quad s_{1} \leq s<s_{2}\end{cases}
$$

where $s_{1}=\cos \theta-\sin \theta$ and $s_{2}=\cos \theta+\sin \theta$.

To obtain $g(\theta, s)$ at values of $\theta$ and $s$ that are not in the region $\{\theta \in[0, \pi / 4], \quad s \geq 0\}$, we use the following symmetry 

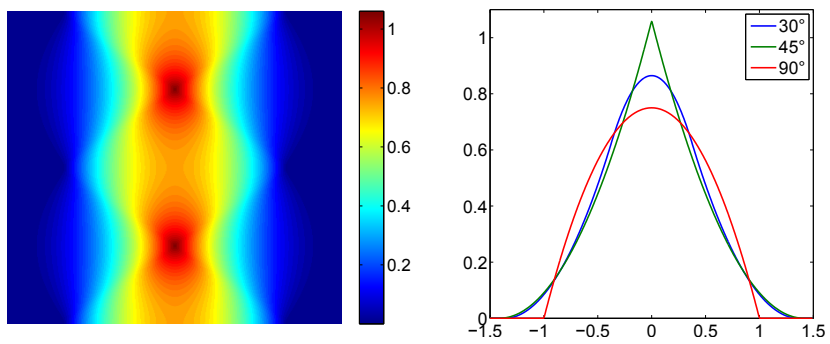

Fig. 2. (left) Sinogram of the pyramid-shaped basis function. (right) Parallelbeam projections at angles $\theta=\pi / 6, \pi / 4, \pi / 2$ (blue, green, red line).

relations:

$$
\begin{array}{ll}
s<0: & g(\theta, s)=g(\theta,-s) \\
\theta \in\left[\frac{\pi}{4}, \frac{\pi}{2}\right]: & g(\theta, s)=g\left(\frac{\pi}{2}-\theta, s\right) \\
\theta \in\left[\frac{\pi}{2}, \frac{3 \pi}{4}\right]: & g(\theta, s)=g\left(\theta-\frac{\pi}{2}, s\right) \\
\theta \in\left[\frac{3 \pi}{4}, \pi\right]: & g(\theta, s)=g(\pi-\theta, s) .
\end{array}
$$

As announced, it can be observed that the Radon transform of the pyramid-shaped basis function is a piecewise quadratic polynomial that is one time continously-differentiable, except for $\theta=m \frac{\pi}{4}$ with $m=0,1, \ldots$ where it is continuous but not differentiable. Fig. 2 illustrates these properties.

\section{EVALUATION METHODOLOGY}

\section{A. Data acquisition and image representation}

Simulations were performed in parallel beam geometry using the FORBILD head phantom. The discretization of the image involved 350 by 350 locations defined with $\Delta x=\Delta y=0.075$. The CT measurements consisted of 1160 views with 380 line integrals per view, defined using a step $\Delta s=0.075$. Each CT measurement was simulated as an average of five line integrals that were calculated each using analytical expressions. The average was introduced to model the finite detector response (disregarding non-linearity effects) and reduce thereby high-frequency errors in the reconstruction process. Then, Poisson noise was added assuming a photon level of 35,000. Altogether, we used 10 noisy data realizations.

We used different grids for the reconstruction: the grid for the pyramid-shaped basis function was shifted by a quarter pixel in $x$ and $y$ relative to the grid for the B-spline basis function.

\section{B. Iterative reconstruction technique}

For reconstruction, the $\mathrm{CT}$ measurements are assumed to be independent normal deviates with equal variances. This assumption is suitable for brain imaging when tube current modulation is applied and the bowtie filter corrects for the shape of the brain. Also, the expected value of the CT measurements is modeled as the Radon transform of $f_{a}$, which can be written as the application of a matrix $A$ to a long vector that groups all coefficients $c_{k, l}$ together.

We seek the maximum likelihood reconstruction, which under our assumptions corresponds to the minimizer of $\|A c-g\|$ where $g$ is the vector grouping the CT measurements. To compute this minimum, we apply the Landweber algorithm, which is given by the formula

$$
c^{(n+1)}=c^{(n)}+\lambda A^{T}\left(g-A c^{(n)}\right)
$$

where $\lambda$ is a convergence controlling factor, chosen as 0.90 times $2 / \sigma_{\max }$, where $\sigma_{\max }$ is the maximum singular value of the projection matrix $A$, estimated using the Power method. After applying a desired number $m$ of iterations, the obtained vector of basis function coefficients $c_{k l}$ is inserted into equation (1) to obtain the expression of the reconstruction.

\section{Image quality}

Image quality was assessed in terms of resolution, bias and noise properties. All bias and noise metrics were computed for the reconstructions obtained every fifth iteration.

1) Resolution: The modulation transfer function (MTF) was used to evaluate resolution. This function was obtained using a phantom that consists only of the central low contrast ellipse within the FORBILD head phantom as well as a phantom that consists only of the left eye within the FORBILD head phantom. For any reconstruction of this phantom, an edge profile that gives the reconstructed value as a function of the distance from the ellipse was computed. Then, the MTF is obtained as the Fourier transform of the differentiated edge profile. Due to our linear reconstruction method, this approach is suitable to evaluate the resolution achieved within the neighborhood of the large low-contrast ellipse and the left eye in reconstructions of the FORBILD head phantom.

Since the resolution varies from one representation to the other and also changes at a different pace for each representation, we present all our figures of merit as a function of the mean MTF value. To obtain the mean MTF value, we computed the area under the MTF curve over the range defined by Nyquist's frequency for the data. This was done for every fifth iteration up to 1000 iterations.

2) Bias: Bias was evaluated as the mean reconstruction error over pixels located within the ellipse and the left eye that is used for the MTF computation. The error for any given pixel was defined as the absolute difference between the reconstructed value and the true attenuation value for this ellipse and the left eye, which is $45 \mathrm{HU}$ and $60 \mathrm{HU}$ respectively.

3) Noise metrics: The image noise was analyzed over a region of interest (ROI) that corresponds to the area within the large, central, low-contrast ellipse and the left eye of the FORBILD head phantom. The analysis relied on the noise magnitude, $\bar{\sigma}$, that was evaluated from pixel variance computations. Using our 10 noise realizations, the pixel variance was first estimated for each pixel location in the ROI. Then, the results were averaged over all pixels in the ROI. The square root of this mean was defined as $\bar{\sigma}$.

\section{IMAGE CORRECTION}

We observed that all reconstructed images with the pyramidshaped basis function display an oblique grid pattern overlaying the structures of interest (see Fig. 4, second image). This may be attributed to the lack of smoothness at 45 degrees (see 
use known input:

1 - water phantom (ellipse)

input:

2 - unknown phantom data
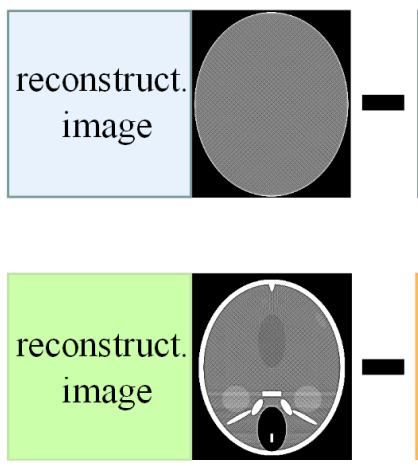

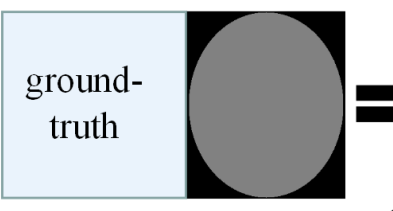

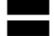

correction

image

correction

image $\square$

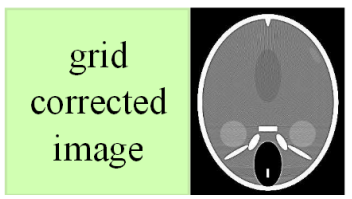

Fig. 3. Two step iterative reconstruction algorithm to correct the reconstructed images obtained with the pyramid-shaped basis function.

\section{B-splines, $\mathrm{n}=0$}
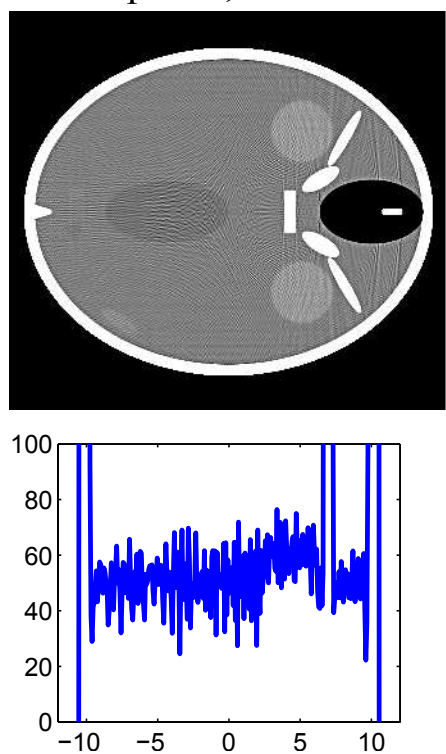

pyramid-shaped basis function not corrected
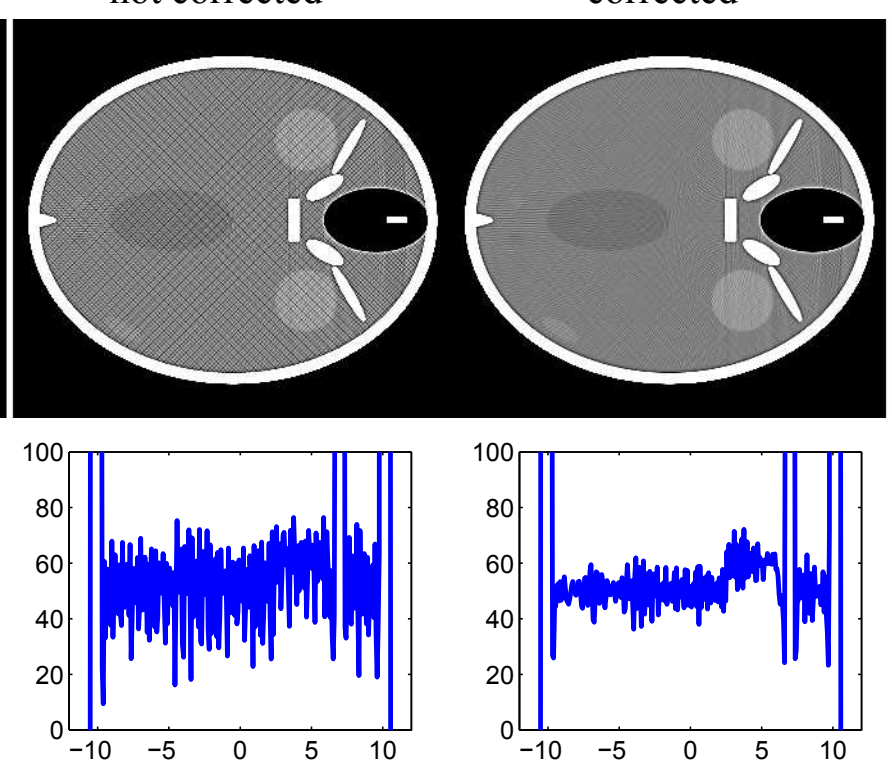

B-splines, $\mathrm{n}=1$
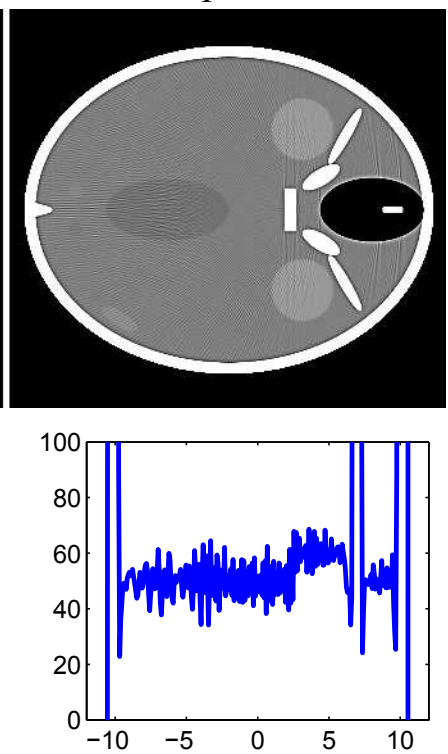

Fig. 4. First row Iterative reconstruction obtained from 300 iterations of the Landweber algorithm: (left) using the B-splines of order $n=0$, (second from left) using the pyramid-shaped basis function with no correction, (third from left) using the pyramid-shaped basis function with correction, (right) using the B-splines of order $n=1$. Grayscale (c/w): 50/100 HU. Second row Horizontal profile through the left (upper) eye for the reconstructions from the row above; the abscissa is is the $\mathrm{y}$-coordinate in $\mathrm{cm}$.
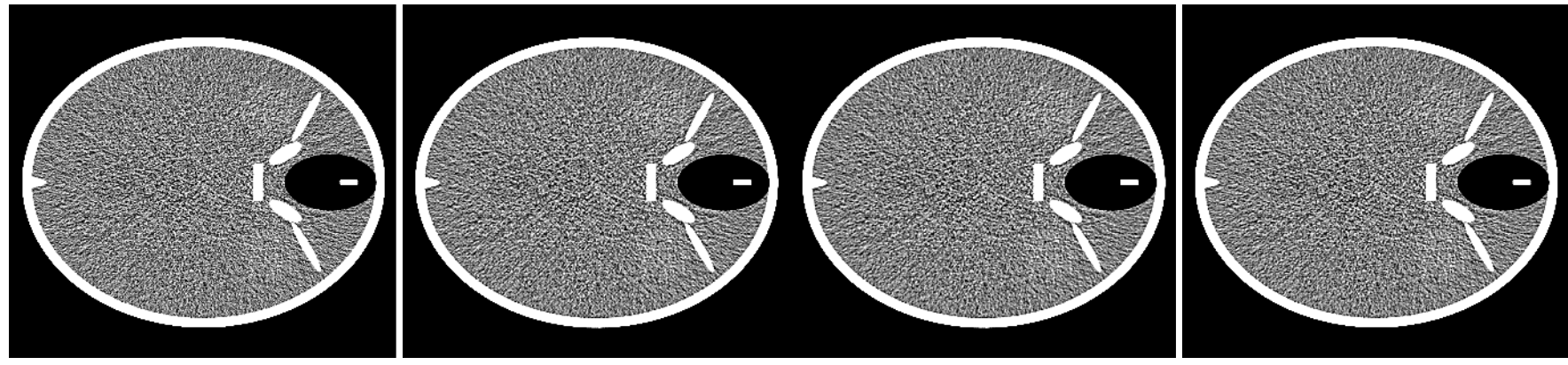

Fig. 5. Iterative reconstruction obtained from 300 iterations of the Landweber algorithm: (left) using the B-splines of order $n=0$, (second from left) using the pyramid-shaped basis function with no correction, (third from left) using the pyramid-shaped basis function with correction, (right) using the B-splines of order $n=1$. Grayscale: $[-50,150] \mathrm{HU}$. 


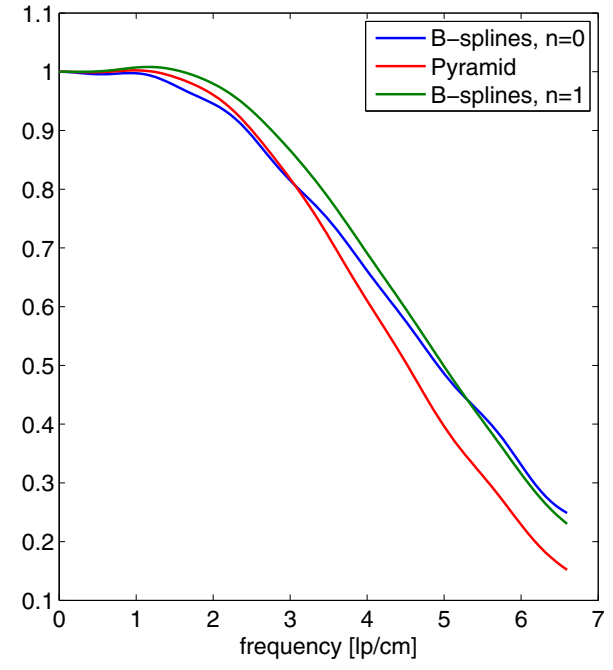

Fig. 6. Modulation transfer function for 300 iterations of the Landweber algorithm using the pyramidshaped basis function and the B-splines of order 0 and 1 .
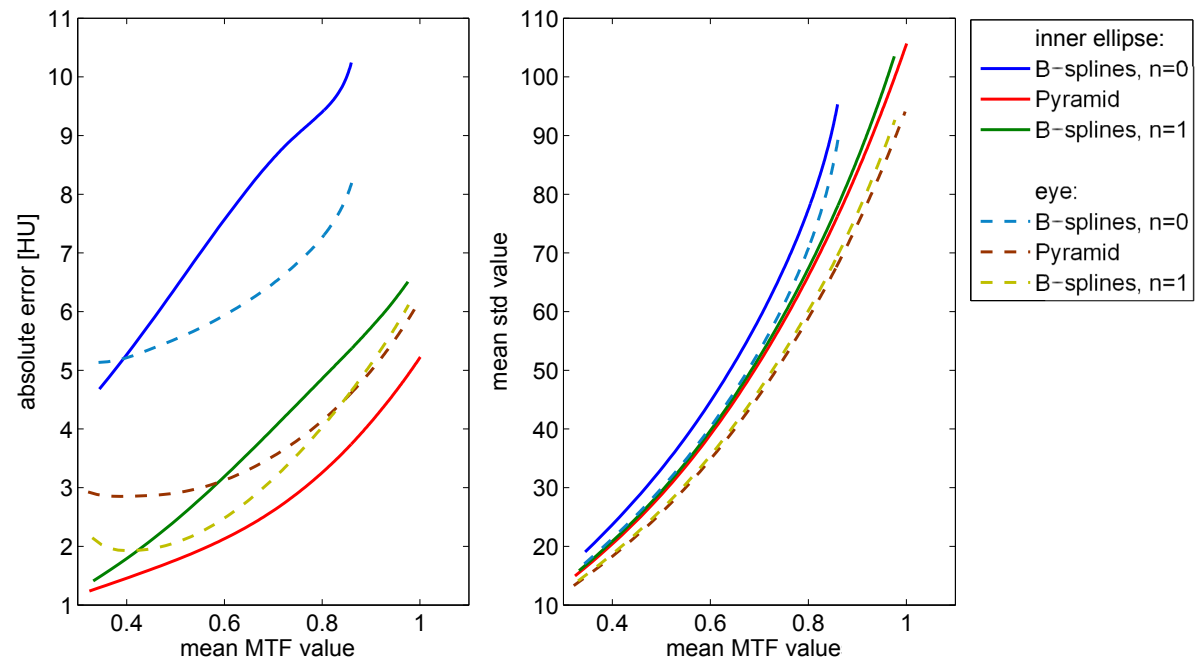

Fig. 7. (left) Mean bias as a function of the mean MTF value obtained by varying the number of iterations by steps of 5 beginning with 50 iterations for the pixels in the central ellipse (solid lines) and the left eye (dashed lines). (right) Spatially-averaged pixel standard deviation $\bar{\sigma}$ evaluated from 10 noise realizations as a function of the mean MTF value.
Fig. 2, right), and may also be due to the basis function partitioning unity only along the rows and columns of the image. We define a two-step iterative algorithm (Fig. 3) to correct the reconstructed images obtained with the pyramid-shaped basis function. In the first step, we introduce an ellipse that encompasses the scanned object, and we apply the iterative reconstruction algorithm to the sinogram of this ellipse. The difference between the resulting reconstruction and the original ellipse defines a correction image which displays only the grid. In the second step, we subtract the correction image from the image reconstructed from the measured data. By applying this two-step iterative algorithm we obtain an image which is nearly free of grid artifacts (Fig. 4, third image). This image was used for the image quality study.

\section{RESUlts}

Figure 4 shows in the first row the results obtained using 300 iterations of the Landweber algorithm by using the B-splines of order $n=0$ (left), using the pyramid-shaped basis function with no correction (second from left), using the pyramidshaped basis function with correction (third from left) and using the B-splines of order $n=1$ (right). The horizontal profile through the left (upper) eye for each reconstruction mentioned above is located below the related image. Fig. 5 shows the reconstructed noisy images in the same arrangement as in Fig. 4. The modulation transfer function for the images in Fig. 4 is shown in Fig. 6. The left hand side of Fig. 7 shows the mean bias as a function of the mean MTF value obtained by varying the number of iterations by steps of 5 beginning with 50 iterations for the pixels in the central ellipse (solid lines) and the left eye (dashed lines). The spatially-averaged pixel standard deviation evaluated from 10 noise realizations as a function of the mean MTF value is shown on the right hand side.

First, we examine, that the modulation transfer function of the B-splines of order $n=0,1$ and the pyramid-shaped basis function show significant differences for the same number of iterations. For that reason, we calculated the mean MTF value as described in section III-C1

Second, for the central low-contrast ellipse the pyramidshaped basis function produce a smaller bias than the B-spline basis functions even if the correction image still show some grid artifacts. For the left eye, the bias curves for the B-splines of order $n=1$ and for the pyramid-shaped basis function intersect each other at 475 iterations. So, for less than 475 iterations we observe that the B-spline basis function produce a smaller bias and for more than 475 iterations a higher bias than the pyramid-shaped basis function. Therefore, depending on the number of iterations the bias also depends on the location of interest.

Third, the images in Fig. 5 show only small differences in noise behavior. This visual impression is confirmed by the plots of the mean standard derivation, $\bar{\sigma}$, which show the same behavior for all basis functions. For example, for the central low-contrast ellipse and for a fixed mean resolution of 0.8 we observe for the B-splines of order $n=0,1$ and for the pyramid-shaped basis function the following values: $\bar{\sigma}_{B s p, n=0}=77.53, \bar{\sigma}_{B s p, n=1}=67.35$ and $\bar{\sigma}_{P y r}=66.10$. Hence, the differences in noise magnitude for the B-splines of order $n=1$ and the pyramid-shaped basis function are tiny $(<1 \mathrm{HU})$, in favor of the pyramid forward projection model.

\section{DISCUSSION}

We have discussed iterative reconstruction using a pyramidshaped basis function that lies at "mid-distance" between the B-splines of order 0 and 1 in the sense that its sinogram is a piecewise quadratic polynomial. We found that this function performs as well as the B-spline of order 1 in terms of bias and noise, with the advantage that the computational effort is lower due to the sinogram having a simpler form. Unfortunately, reconstruction with the pyramid-shaped basis function requires a correction method to remove an inconvenient grid pattern. 
This correction was shown to work satisfactorily for brain imaging but its applicability to other anatomical regions such as the thorax has not been tested.

\section{REFERENCES}

[1] M. U. S. Horbelt, M. Liebling, "Discretization of the radon transform and of its inverse by spline convolutions," IEEE Transactions on Medical Imaging, vol. 21, pp. 363-376, 2002.

[2] R. M. Lewitt, "Multidimensional digital image representations using generalized kaiser-bessel window functions," J. Opt. Soc. am. a, vol. 7, pp. 1834-46, 1990. 